\title{
Ground State Properties of Rock Salt, CsCl, Diamond and Zinc Blende Structured Solids
}

\author{
R.K. Singh ${ }^{1}$, A.S. Verma ${ }^{*}, 2$ and S.K. Rathi ${ }^{1}$ \\ ${ }^{I}$ Department of Physics, B. S. A. College, Mathura, 281004, India \\ ${ }^{2}$ Department of Physics, Sanjay Institute of Engineering and Management, Mathura, 281406, India
}

\begin{abstract}
In this paper we extend to rock salt, $\mathrm{CsCl}$, diamond and zinc blende structured solids with conduction $\mathrm{d}$ electrons the calculation of ground state properties such as bulk modulus and cohesive energy using the plasmon oscillations theory of solids formalism already employed for chalcopyrite semiconductors. The present method is not limited to binary or ternary compounds but can be use for all the semi-conducting and insulating compounds. The calculated values are in excellent agreement with the observed and calculated values of different researchers.
\end{abstract}

Keywords: Bulk modulus, cohesive energy and plasmon energy.

\section{INTRODUCTION}

In recent years increasing attention has been given towards the study of rock salt, $\mathrm{CsCl}$, diamond and zinc blende structured solids. Alkali halides and alkaline-earth chalcogenides have attracted attention of physicists due to many practical uses and rock-salt crystal structures. Alkaline earth chalcogenides are important in geophysical science and research since they are link between the highly ionic alkali halides and covalent III-V compounds [1]. According to the classical valence picture, all the elements from column IV of the periodic table $(\mathrm{C}, \mathrm{Si}, \mathrm{Ge}$ and $\mathrm{Sn})$ should be capable of forming diamond-type crystals. Each atom has four valence electrons, which occupy the tetrahedrally hybridised $\mathrm{sp}^{3}$ orbitals pointing towards the four nearest neighbours and this is true for C through Sn. Most of the semiconductors that are used in the modern microelectronic industry have the zinc blende crystallographic structure. The crystals with zinc blende or sphalerite structure range from raw iron and zinc minerals to man-made $\mathrm{GaN}$ and $\mathrm{BN}$ semiconductors. The particular omnitriangulated nature in atomic structure gives these materials unique physical properties. Recently [2-6], there is an attractive considerable interest on II-VI semiconductors and their alloys due to their application in photovoltaic devices, as electro-optic crystals with ultra fast response time spectroscopy and recent realization of lightemitting diodes (based on ZnTe). This has motivated several theorists to modelling band offset [7], structural and thermo dynamical properties in this family and their alloys $[8,9]$ and to revise many fundamental physical properties based on $a b$ initio calculations [10]. In fact, the role of metal d-states in II-VI semiconductors is very important. Wei and Zunger [11], have shown that $p-d$ repulsion and hybridisation (i) lower the band gaps, (ii) reduce the cohesive energy, (iii)

*Address correspondence to this author at the Department of Physics, Sanjay Institute of Engineering and Management, Mathura, 281406, India; Tel: +91 565 2423417; E-mail: ajay_phy@rediffmail.com increase the equilibrium lattice parameters and also have an influence on other features.

A considerable amount of experimental and theoretical work has been done during the last few years on the bulk modulus and cohesive energies of these semiconductors and insulators. Modern computational methods have made it possible to study the structural, mechanical and optical properties of a wide variety of molecules and solids in great detail. There are however, instances where this level of detail either cannot be easily attained because of the complexity of the system or is not needed, as when studying broad trends in the behaviour of a large set of systems. Empirical concepts such as valence, empirical radii, electronegativity, ionicity and plasmon energy are then useful $[12,13]$. These concepts are directly associated with the character of the chemical bond and thus provide means for explaining and classifying many basic properties of molecules and solids.

Therefore, we thought it would be of interest to give an alternative explanation for bulk modulus and cohesive energies of rock salt, $\mathrm{CsCl}$, diamond and II-VI zinc blende structured solids. In this paper we propose a method based on a plasma oscillations theory of solids for the calculation of the bulk modulus ( $\mathrm{B}$ in $\mathrm{GPa}$ ), and cohesive energy ( $\mathrm{E}_{\mathrm{coh}}$ in $\mathrm{kcal} / \mathrm{mol}$ ) of these semiconductors and insulators. It is now well established that the plasmon energy of a metal changes $[14,15]$, when it undergoes a chemical combination and forms a compound. A plasmon is a collective excitation of the conduction electrons in a metal with energy, $\hbar \omega_{\mathrm{p}}$, which depends on the density of the conduction electrons. This is due to the fact that the plasmon energy depends on the density of the conduction electrons and effective number of valence electrons, which changes when a metal forms a compound. We have calculated the bulk modulus (B in $\mathrm{GPa})$, and cohesive energy $\left(\mathrm{E}_{\mathrm{coh}}\right.$ in $\left.\mathrm{kcal} / \mathrm{mol}\right)$ in rock salt, $\mathrm{CsCl}$, diamond and II-VI zinc blende structured solids using this idea. 
The present paper is an extension of our earlier work [16], where the mechanical and optical properties of $\mathrm{A}^{\mathrm{I}} \mathrm{B}^{\mathrm{III}} \mathrm{C}_{2}{ }^{\mathrm{VI}}$ and $\mathrm{A}^{\mathrm{II}} \mathrm{B}^{\mathrm{IV}} \mathrm{C}_{2}{ }^{\mathrm{V}}$ semiconductors of the chalcopyrite structural type were quantitatively calculated using plasmon oscillations theory of solids, and we have shown that this theory is a widely applicable method in calculating or predicting mechanical and optical properties such as microhardness, bulk modulus, dielectric constant, electronic polarizability and susceptibility of crystal materials. However, one of the most important aspects of this method is that the detailed crystal structures of the materials to be studied must be clearly known.

\section{THEORETICAL CONCEPTS}

A metallic crystal may be described as an assembly of immediate positive ion cores and conduction electrons that are nearly free to move over the whole of the crystal. The condition of charge neutrality is maintained because of the balance struck between the negative charge on conduction electrons and an equal concentration of positive charge on ion cores. Thus a metal serves as a good example of plasma. A random motion may be a momentary fluctuation in the equilibrium position of an electron, caused by the average electrostatic field of all other electrons. The position fluctuation would create a charge imbalance in the region of that electron, as a result of which other electrons would rush into that region in order to restore the condition of charge neutrality. At any finite temperature, electrons being very light particles move with fairly large speed relative to ions which we consider to be at rest. The electrons rushing into the region of the electron that suffered a fluctuation in its equilibrium position are unable to stop at the desired positions and overshoot their mark on account of the large kinetic energy which by the way represents the total energy there. As soon as the energy goes totally electrostatic, electrons turn around and attempt again to approach the wanted locations in the region of the misbehaving electron. The repetition of this process constitutes the collective oscillatory motion. These collective oscillations are called plasma oscillations. The energy of a quantum of plasma oscillations of the valence electrons in both metal and compound is given by the relation [17],

$\hbar \omega_{\mathrm{p}}=28.8 \sqrt{ }(\mathrm{Z} \sigma / \mathrm{W})$

where $\mathrm{Z}$ is the number of electrons taking part in the plasma oscillations, $\sigma$ the specific gravity and $\mathrm{W}$ the molecular weight. Equation (1) is valid for free electrons but it is also applicable for semiconductors and insulators, up to a first approximation. Raether [18], and Philipp and Ehrenreich [19] have shown that the plasmon energy for semiconductors and insulators is given by,

$\hbar \omega_{\mathrm{pd}}=\hbar \omega_{\mathrm{p}} /\left(1-\delta \varepsilon_{\mathrm{o}}\right)^{1 / 2}$

where $\delta \varepsilon_{0}$ is a very small correction to the free-electron plasmon energy $\hbar \omega_{p}$ and can be neglected to a first approximation. Philipp and Ehrenreich [19] have shown that the calculated values of $\hbar \omega_{\mathrm{p}}$ and $\hbar \omega_{\mathrm{pd}}$ are in fair agreement with their observed values of plasmon energy in dielectrics. It has also been pointed out by Kittel [20] that the plasmon oscillations in dielectrics are physically the same as in metals.

Presently, Srivastava [21-23] has studied electronic properties for rock salt, zinc blende and $\mathrm{CsCl}$ structured solids in terms of plasmon energy $\left(\hbar \omega_{p}\right)$ by the following relations,

$\mathrm{E}_{\mathrm{h}}=\mathrm{K}_{1}\left(\hbar \omega_{\mathrm{p}}\right)^{\mathrm{v}} \mathrm{eV}$,

$\mathrm{E}_{\mathrm{c}}=\mathrm{K}_{2} \mathrm{~b}\left(\hbar \omega_{\mathrm{p}}\right)^{2 / 3} \times \exp \left[-\mathrm{K}_{3}\left(\hbar \omega_{\mathrm{p}}\right)^{-1 / 3}\right] \mathrm{eV}$

$\mathrm{d}=\mathrm{K}_{4}\left(\hbar \omega_{\mathrm{p}}\right)^{-2 / 3}\left(\hbar \omega_{\mathrm{p}}\right.$ in $\mathrm{eV}, \mathrm{d}$ in $\AA$ )

Here $E_{h}, E_{c}$ and $d$ are homopolar gaps, ionic gaps and bond length respectively, $\mathrm{K}_{1}, \mathrm{~K}_{2}, \mathrm{~K}_{3}, \mathrm{~K}_{4}$ and $v$ are constants depending upon the structure elements and $\mathrm{b}$ is prescreening factor. Recently, Kumar et al. [13], have shown that heat of formation for $\mathrm{A}^{\mathrm{I}} \mathrm{B}^{\mathrm{III}} \mathrm{C}_{2}{ }^{\mathrm{VI}}$ and $\mathrm{A}^{\mathrm{II}} \mathrm{B}^{\mathrm{IV}} \mathrm{C}_{2}{ }^{\mathrm{V}}$ semiconductors may be determine in terms of plasmon energy by following form,

Heat of formation $=\mathrm{C}\left(\hbar \omega_{\mathrm{p}}\right)^{\mathrm{C}^{\prime}}$

where $\mathrm{C}$ and $\mathrm{C}^{\prime}$ are constants.

In previous work [16], we have evaluated mechanical and optical properties such as microhardness $(\mathrm{H})$, bulk modulus $(B)$, dielectric constant $(\varepsilon)$, electronic polarizability $(\alpha)$ and susceptibility $(\chi)$ for chalcopyrites in terms of plasmon energy $\hbar \omega_{\mathrm{p}}\left(\hbar \omega_{\mathrm{p}}\right.$ in $\left.\mathrm{eV}\right)$, for $\mathrm{A}^{\mathrm{II}} \mathrm{B}^{\mathrm{IV}} \mathrm{C}_{2} \mathrm{~V}$

Microhardness $(\mathrm{H})=0.001\left(\hbar \omega_{\mathrm{p}}\right)^{4.89}$

for $\mathrm{A}^{\mathrm{I}} \mathrm{B}^{\mathrm{III}} \mathrm{C}_{2}{ }^{\mathrm{VI}}$

Microhardness $(\mathrm{H})=0.192\left(\hbar \omega_{\mathrm{p}}\right)^{2.331}$

for $\mathrm{A}^{\mathrm{I}} \mathrm{B}^{\mathrm{III}} \mathrm{C}_{2}{ }^{\mathrm{VI}}$ and $\mathrm{A}^{\mathrm{II}} \mathrm{B}^{\mathrm{IV}} \mathrm{C}_{2}{ }^{\mathrm{V}}$

Bulk modulus $(\mathrm{B})=0.005\left(\hbar \omega_{\mathrm{p}}\right)^{\mathrm{S}}$

for $\mathrm{A}^{\mathrm{I}} \mathrm{B}^{\mathrm{III}} \mathrm{C}_{2}{ }^{\mathrm{VI}}$ and $\mathrm{A}^{\mathrm{II}} \mathrm{B}^{\mathrm{IV}} \mathrm{C}_{2}{ }^{\mathrm{V}}$

Dielectric constant $(\varepsilon)=500 /\left(\hbar \omega_{\mathrm{p}}\right)^{\mathrm{V}}$

for $\mathrm{A}^{\mathrm{I}} \mathrm{B}^{\mathrm{III}} \mathrm{C}_{2}{ }^{\mathrm{VI}}$ and $\mathrm{A}^{\mathrm{II}} \mathrm{B}^{\mathrm{IV}} \mathrm{C}_{2}{ }^{\mathrm{V}}$

Polarizability $(\alpha)=3500 /\left(\hbar \omega_{\mathrm{p}}\right)^{\mathrm{D}}$

where $\mathrm{S}, \mathrm{V}$ and $\mathrm{D}$ are constants, which depends upon group of materials. Similarly, based on the above expressions, we are of the view that some parameters related to ground state properties (i.e. bulk modulus and cohesive energy) of rock salt, $\mathrm{CsCl}$, diamond and II-VI zinc blende structured solids can be evaluated using their plasmon energy ( $\hbar \omega_{\mathrm{p}}$ in $\mathrm{eV}$ ) by following relations,

Bulk modulus $(\mathrm{B}$ in $\mathrm{GPa})=\mathrm{A}\left(\hbar \omega_{\mathrm{p}}\right)^{\mathrm{A}^{\prime}}$

and

Cohesive energy $\left(\mathrm{E}_{\mathrm{coh}}\right.$ in $\left.\mathrm{kcal} / \mathrm{mol}\right)=\mathrm{S}\left(\hbar \omega_{\mathrm{p}}\right)^{\mathrm{S}^{\prime}}$

where $\mathrm{A}, \mathrm{A}^{\prime}, \mathrm{S}$ and $\mathrm{S}^{\prime}$ are the numerical constants depending upon the structure element (i.e. group of materials). These 
values are $\mathrm{A}^{\prime}=2$ for rock salt, $\mathrm{CsCl}$ and zinc blende crystals, $\mathrm{A}^{\prime}=2.4$ for diamond crystals, $\mathrm{S}^{\prime}=0.56$ for rock salt and $\mathrm{CsCl}$ crystals and $\mathrm{S}^{\prime}=0.99$ for zinc blende and diamond crystals. The values of A and S are listed in Table $\mathbf{1 .}$

Table 1. Value of Constants

\begin{tabular}{|l|c|c|}
\hline \multicolumn{1}{|c|}{ Materials } & A & S \\
\hline \hline Alkali halides and CsCl crystals & 0.1 & 39.88 \\
\hline Alkaline-earth chalcogenides & 0.282 & 165 \\
\hline zinc blende crystals & 0.275 & 7.75 \\
\hline diamond crystals & 0.115 & 10.75 \\
\hline
\end{tabular}

\section{RESULTS AND DISCUSSION}

The bulk modulus and cohesive energy are important ground state properties of a material; bulk modulus defines its resistance to volume change when compressed. Both experimental and theoretical results suggest that the bulk modulus reflects the hardness of the materials [24, 25]. Jayaraman et al. [26] predicted that the bulk modulus directly depends on the product of the ionic charges. Any change in crystallographic environment of an atom is related to core electrons via the valence electrons. The change in wave function that occurs for the outer electrons usually means a displacement of electric charge in the valence shell so that the interaction between valence, shell, and core electrons is changed. This leads to a change in binding energy of the inner electron and to a shift in the position of the absorption edge. plasmon energy also depends on the effective number of valence electrons and density of the conduction electrons, which changes when a metal forms a compound.

According to this idea, we have proposed relations (4) and (5) for the calculation of bulk modulus and cohesive energy of alkali halides, cuprous halides, thallous halides, alkaline-earth chalcogenides, group IV and II-VI semiconductors. The values so obtained are presented in the following Tables $\mathbf{2}$ and $\mathbf{3}$ compared with the experimental and theoretical data reported so far. For example the results for bulk modulus differ from experimental by, $\mathrm{LiCl}-0 \%, \mathrm{C}-$ $0 \%, \mathrm{Si}-0 \%$ and $\mathrm{ZnS}-0 \%$, and the results for cohesive energy differ from experimental by $\mathrm{LiF}-0 \%, \mathrm{KF}-0 \%, \mathrm{KCl}-0 \%$, $\mathrm{KBr}-0 \%, \mathrm{KI}-0 \%, \mathrm{RbBr}-0 \%$ and $\mathrm{RbI}-0 \%$ in the current study. We note that proposed approach is quite reasonable and can give us a useful guide in calculating and predicting the bulk modulus and cohesive energy of crystal materials. Thus it is possible to predict the order of bulk modulus and cohesive energies of semi conducting and insulating compounds from their plasmon energies. The present approach for the calculation of the ground state properties of solids can be used for semi conducting and insulating compounds without having knowledge of crystal ionicity, transition pressure, while the model given by Neumann [27], and Al-Douri et al. [28] requires the values of these parameters to calculate the bulk modulus of solids and is limited to the simple binary compounds.

\section{CONCLUSION}

From the above results obtained by using the proposed approach, it is quite obvious that the parameters such as bulk modulus (B in GPa), and cohesive energy $\left(E_{\text {coh }}\right.$ in $\left.\mathrm{kcal} / \mathrm{mol}\right)$ reflecting the ground state properties, can be expressed in terms of plasmon energies of these materials, which is definitely a surprising phenomenon. The calculated values are presented in Tables $\mathbf{2}$ and 3. We note that calculated values are in close agreement with values determined directly from experiment. The various parameters evaluated in this work hardly deviates $0 \%$ to $6 \%$ and the maximum discrepancy for bulk modulus is $9 \%$ in the case of $\mathrm{Ge}$ from the experimental data. The values evaluated show a systematic trend and are consistent with the available data reported so far, which proves the validity of the approach. Since we have been reasonably successful in calculating these parameters using the plasmon frequency of the materials for chalcopyrite crystals (A. S. Verma et al. [16]), it is natural to say that this approach can easily be extended to rock salt, $\mathrm{CsCl}$, diamond and zinc blende crystals. Hence it is possible to predict the order of ground state properties of semiconducting and insulating compounds from their plasmon energies.

Table 2. In this Table we have Presented Theoretical (Theo.), Experimental (Exp.) and Calculated Values of Cohesive Energy (E in $\mathrm{kcal} / \mathrm{mol}$.) and Isothermal Bulk Modulus (B in GPa) for CsCI Structured Solids

\begin{tabular}{|c|c|c|c|c|c|c|}
\hline Solids & $\hbar \omega_{p}^{[23]}$ & $\mathbf{E}_{\text {coh. }}$ theo. [29] & $\mathbf{E}_{\text {coh. }}{ }^{\text {exp. [29] }}$ & $\mathbf{E}_{\text {coh. }}{ }^{\text {This work }}$ & $\mathbf{B}^{\text {theo. [30] }}$ & $\mathbf{B}^{\text {This work }}$ \\
\hline $\mathrm{CsCl}$ & 12.51 & 166 & 160 & 164 & 16 & 16 \\
\hline $\mathrm{CsBr}$ & 11.77 & 163 & 154 & 159 & 14 & 14 \\
\hline CsI & 10.73 & 151 & 146 & 151 & 11 & 12 \\
\hline $\mathrm{TlBr}$ & 13.28 & 194 & 169 & 170 & 21 & 18 \\
\hline TlI & 11.92 & & & 160 & 17 & 14 \\
\hline
\end{tabular}


Table 3. In this Table we have Presented Theoretical (Theo.), Experimental (Exp.) and Calculated Values of Cohesive Energy ( $E_{\text {coh }}$ in kcal/mol.) and Isothermal Bulk Modulus (B in GPa) for Alkali Halides and Alkaline Earth Chalcogenides, Group IV and II-VI Semiconductors

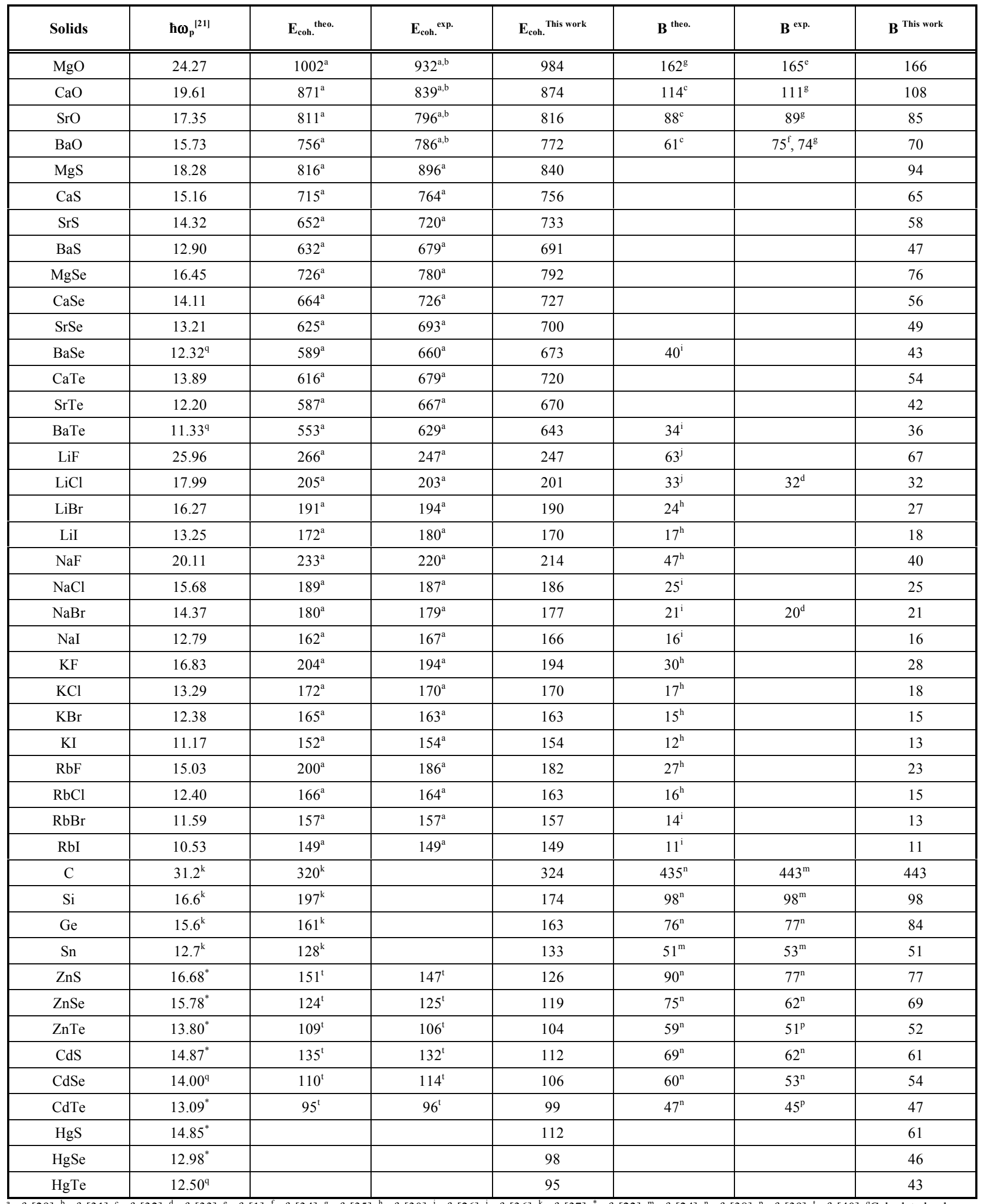




\section{REFERENCES}

[1] Alfe D. Melting curve of $\mathrm{MgO}$ from first-principles simulations. Phys Rev Lett 2005; 94: 235701-4.

[2] Contreras-Puente G, Vigil O, Ortega-Lopez M, Morales-Acevedo A, Vidal J, Albor-Aguilera ML. New window materials used as heterojunction partners on CdTe solar cells. Thin Solid Films 2000; 361: 378-82.

[3] Nahata A, Auston DH, Heinz TF, Wu C. Coherent detection of freely propagating terahertz radiation by electro-optic sampling. Appl Phys Lett 1996; 68: 150-2.

[4] Knoesel E, Bonn M, Shan J, Heinz TF. Charge transport and carrier dynamics in liquids probed by $\mathrm{THz}$ time-domain spectroscopy. Phys Rev Lett 2001; 86: 340-3.

[5] Shall M, Walther M, Uhd Jepsen P. Fundamental and second-order phonon processes in CdTe and ZnTe. Phys Rev B 2001; 64: 094301-8.

[6] Sato K, Hanafusa M, Noda A, Arakawa A, Uchida M, Asahi T, Oda $\mathrm{O}$. ZnTe pure green light-emitting diodes fabricated by thermal diffusion. J Cryst Growth 2000; 214/215: 1080-4.

[7] Unlu H. Modelling of band offsets in II-VI heterostructures. Phys Stat Sol B 2002; 229: 581-5.

[8] Merad A E, Aourag H, Khelifa B, Mathieu C, Merad G. Predictions of the bonding properties inCd $\mathrm{d}_{1-\mathrm{x}} \mathrm{Zn}_{\mathrm{x}}$ Te. Superlatt Microstruct 2001; 30: 241-51.

[9] Kanoun MB, Merad AE, Aourag H, Cibert J, Merad G. Moleculardynamics simulations of structural and thermodynamic properties of ZnTe using a three-body potential. Solid State Sci 2003; 5: 1211-16.

[10] Wei SH, Zunger A. Predicted band-gap pressure coefficients of all diamond and zinc-blende semiconductors: Chemical trends. Phys Rev B 1999; 60: 5404-11.

[11] Wei SH, Zunger A. Role of metal d states in II-VI semiconductors. Phys Rev B 1988; 37: 8958-81.

[12] Pauling L. The Nature of the Chemical Bond, $3^{\text {rd }}$, ed. Cornell University Press: Ithaca; 1960.

[13] Kumar V, Sastry BSR. Heat of formation of ternary chalcopyrite semiconductors. J Phys Chem Solids 2005; 66: 99-102.

[14] Srivastava KS, Shrivastava RL, Harsh OK, Kumar V. Low-energy plasmon $\mathrm{K} \beta^{\prime}$ satellite in the $\mathrm{K} \beta_{1,3} \mathrm{X}$-ray emission spectra of $\mathrm{Mn}, \mathrm{Cr}$, and their compounds. Phys Rev B 1979; 19: 4336-9.

[15] Srivastava KS, Shrivastava RL, Harsh OK, Kumar V. X-ray Kabsorption edge shifts in transition elements. J Phys Chem Solids 1979; 40: 489-91.

[16] Verma AS, Bhardwaj SR. Mechanical and optical properties of $\mathrm{A}^{\mathrm{II}} \mathrm{B}^{\mathrm{IV}} \mathrm{C}_{2}{ }^{\mathrm{V}}$ and $\mathrm{A}^{\mathrm{I}} \mathrm{B}^{\mathrm{III}} \mathrm{C}_{2}{ }^{\mathrm{VI}}$ semiconductors. Phys Stat Sol B 2006; 243: 2858-63.

[17] Marton L, Leder LB, Mendlowitz H. Advances in Electronics and Electron Physics. In: Marton L, Ed, Academic Press, New York, 1955; vol. 7: pp. 225.

[18] Raether H. Tracts in Modern Physics, Ergebnisse der exakten Naturwissenschaften. In: G. Höhler, Ed, Springer, Berlin, 1965; vol. 34, pp. 84 .
[19] Philipp HR, Ehrenreich H. Optical properties of semiconductors. Phys Rev 1963; 129: 1550-60.

[20] Kittel C. Introduction to Solid State Physics, $4^{\text {th }}$ ed. Wiley: New York; 1971, Second Wiley Eastern Reprint: New Delhi, 1974; pp. 227.

[21] Srivastava VK. Homopolar and heteropolar energy gaps of an ionic system. Phys Rev B 1984; 29: 6993-5.

[22] Srivastava VK. Homopolar and heteropolar energy gaps in zincblende crystals. J Phys C: Solid State Phys 1986; 19: 5689-94.

[23] Srivastava VK. Ionic and covalent energy gaps of $\mathrm{CsCl}$ crystals. Phys. Lett A 1984; 102: 127-9.

[24] Wang SQ, Ye HQ. Plane-wave pseudopotential study on mechanical and electronic properties for IV and III-V crystalline phases with zinc-blende structure. Phys Rev B 2002; 66: 235111-7.

[25] Al-Douri Y, Abid H, Aourag H. Correlation between the bulk Modulus and the charge density in semiconductors. Physica B 2001; 305: 186-90

[26] Jayaraman A, Batlogg B, Maines RG, Bach H. Effective ionic charge and bulk modulus scaling in rocksalt-structured rare-earth compounds. Phys Rev B 1982; 26: 3347-51.

[27] Neumann H. Bulk modulus - volume relationship in alkali halides with rocksalt structure. Crystal Res Technol 1988; 23: 531-6.

[28] Al-Douri Y, Abid H, Aourag A. Correlation between the bulk modulus and the transition pressure in semiconductors. Materials Lett 2005; 59: 2032-4.

[29] Gupta VP, Sipani SK. Cohesive energy of ionic crystals within the framework of compressible ion theory. Phys Stat sol B 1982; 111: 295-301.

[30] Schlosser H. Cohesive energy-lattice constant and bulk moduluslattice constant relationships: Alkali halides, Ag halides, Tl halides. J Phys Chem Solids 1992; 53: 855-6.

[31] Schlosser H. Cohesive energy-lattice constant relationship. Phys Status Solidi B 1993; 179: k1-k3.

[32] Sirdeshmukh DB, Subhadra KG. Bulk modulus-volume relationship for some crystals with a rock salt structure. J Appl Phys 1986; 59: 276-7.

[33] Fang ZH, Chen LR. The search for a universal equation of state and the bulk modulus for solids. Phys Stat Sol B 1993; 180: k5-k10.

[34] Mota RC, Costa SC, Pizani PS, Rino JP. Molecular dynamics simulation of the structural and dynamical properties of crystalline BaO. Phys Rev B 2005; 71: 224114-20.

[35] Gupta BRK, Goyal RP. Static and thermophysical properties of chalcogenide crystals with $\mathrm{NaCl}$ structure. Solid State Commun 1984; 49: 559-62.

[36] Kumari M, Dass N. An equation of state applied to 50 solids. II. J Phys Condens Matter 1990; 2: 7891-5.

[37] Phillips JC. Bonds and Bands in Semiconductors. Acadamic Press Inc., London, 1973; pp. 29, 49, 52

[38] Cohen ML. Calculation of bulk moduli of diamond and zinc-blende solids. Phys Rev B 1985; 32: 7988-91.

[39] Merad AE, Kanoun MB, Merad G, Cibert J, Aourag H. Fullpotential investigation of the electronic and optical properties of stressed CdTe and ZnTe. Mater Chem Phys 2005; 92: 333-9.

[40] Aresti A, Garbato L, Rucci A. Some cohesive energy features of tetrahedral semiconductors. J Phys Chem Solids 1984; 45: 361-5. 